\title{
CORRESPONDENCE
}

\section{Population-specific reference equations?}

\section{To the Editors:}

The recent paper by CHINN et al. [1], published in the European Respiratory Journal, describing sources of variation of forced expiratory volume in one second (FEV1) and forced vital capacity in the large multicentre European Community Respiratory Health Survey trial, raises important issues in the ongoing debate over the misuse of reference equations in respiratory medicine. The authors demonstrate potentially important population differences, which call into question the application of ethnicity-specific reference equations.

However, we believe it is likely that much of the population heterogeneity in the study by CHINN et al. [1] arose from differences in methods, equipment and software. While the authors made some attempts to account for such differences, the level of "noise" and the limited site-specific details available (such as quality-control criteria and software versions) would make it hard to demonstrate statistically significant contributions from all sources.

Although the differences were not explained by the different equipment models, differences in equipment software version or measurement protocols could have led to systematic measurement errors. Since quality-control visits were conducted as part of the study protocol, it may have been reasonable to measure biological controls in each of the centres to assess the intra-subject variability between test centres [2]. As the authors suggest, a multicentre trial with a common protocol and identical equipment and quality-control techniques may be better able to tease apart methodological and equipment differences from true population differences.

Given the strong correlation between height and FEV1, it is remarkable that a quarter of the centres did not measure height. Self-reported height is known to be overestimated, especially in males [3-5], and this could have contributed significantly to the observed inter-centre variability.

Furthermore, while the ethnicity of the study population was stated to be "almost exclusively White", it is unclear whether, in countries with multi-ethnic populations, such as the UK or the USA, non-Caucasians were excluded or merely studied in small numbers. Inclusion of even a small number of non-White subjects could increase the variability of spirometry measurements. Also, the recruitment of an exclusively White sample may be unrepresentative of the overall population owing to other confounders.

The study by CHINN et al. [1] raises awareness of the misuse of reference equations, but offers no realistic alternative. In the meantime, individual laboratories should take every precaution to ensure the reference equations installed in their equipment are appropriate for the population being studied. The inter-centre differences in the European Community
Respiratory Health Study cannot be ignored. Current reference equations cannot be guaranteed to give accurate norms of lung health and this study emphasises the need for more suitable reference equations and/or statistical models that can adjust for between-centre differences.

\author{
S. Stanojevic, A. Wade, T.J. Cole and J. Stocks \\ UCL, Institute of Child Health, London, UK.
}

\section{REFERENCES}

1 Chinn S, Jarvis D, Svanes C, Burney P. Sources of variation in forced expiratory volume in one second and forced vital capacity. Eur Respir J 2006; 27: 767-773.

2 Beardsmore CS, Paton J, Thompson JR, et al. Standardized lung function laboratories for multicenter trials. Pediatr Pulmonol 2006; (In press).

3 Niedhammer I, Bugel I, Bonenfant S, Goldberg M, Leclerc A. Validity of self-reported weight and height in the French GAZEL cohort. Int J Obes Relat Metab Disord 2000; 24: 1111-1118.

4 Stewart AW, Jackson RT, Ford MA, Beaglehole R. Underestimation of relative weight by use of self-reported height and weight. Am J Epidemiol 1987; 125: 122-126.

5 Flegal KM, Carroll MD, Ogden CL, Johnson CL. Prevalence and trends in obesity among US adults, 1999-2000. JAMA 2002; 288: 1723-1727.

DOI: 10.1183/09031936.00119606

\section{From the authors:}

We thank S. Stanojevic and co-workers for their letter and the opportunity it gives us to provide some clarification.

Although our paper [1] is recent, the data were collected in the European Community Respiratory Health Study (ECRHS) I, carried out from 1990-1992, by research teams who had not worked together previously and who came from diverse healthcare systems and different language groups, across four continents.

We are well aware of improvements and clarifications that could have been made to the written protocol and to training and quality-control procedures. Unfortunately, we do not have access to the "in press" paper [2] referred to in the letter from S. Stanojevic and co-workers. While biological controls may have some advantages for assessing between-centre variations, it is not at all clear to us how many would be needed and what the effects of training would be. However, almost certainly the number of biological controls that would have been needed to assess intra-subject variability between centres would not have been available. 